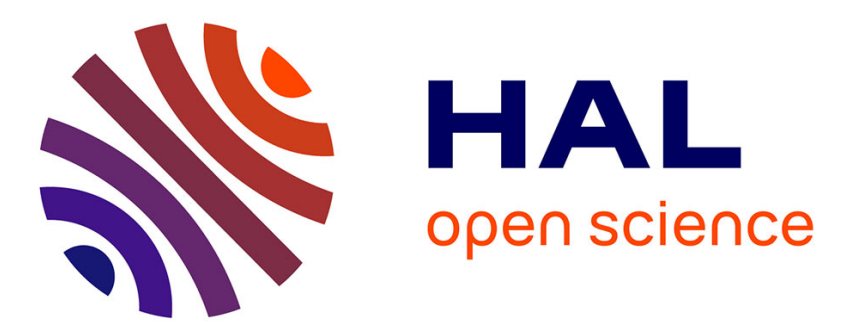

\title{
Impact of Interferences on Bandwidth Reservation for Ad Hoc Networks: a First Theoretical Study
}

Karell Bertet, Claude Chaudet, Isabelle Guérin-Lassous, Laurent Viennot

\section{To cite this version:}

Karell Bertet, Claude Chaudet, Isabelle Guérin-Lassous, Laurent Viennot. Impact of Interferences on Bandwidth Reservation for Ad Hoc Networks: a First Theoretical Study. IEEE Conference and Exhibition on Global Telecommunications (GLOBECOM), Nov 2001, San Antonio, United States. pp.2907 - 2910, 10.1109/GLOCOM.2001.965960 . inria-00471697

\section{HAL Id: inria-00471697 https://hal.inria.fr/inria-00471697}

Submitted on 8 Apr 2010

HAL is a multi-disciplinary open access archive for the deposit and dissemination of scientific research documents, whether they are published or not. The documents may come from teaching and research institutions in France or abroad, or from public or private research centers.
L'archive ouverte pluridisciplinaire HAL, est destinée au dépôt et à la diffusion de documents scientifiques de niveau recherche, publiés ou non, émanant des établissements d'enseignement et de recherche français ou étrangers, des laboratoires publics ou privés. 


\title{
Impact of Interferences on Bandwidth Reservation for Ad Hoc Networks: a First Theoretical Study
}

\author{
K. Bertet, C. Chaudet, I. Guérin Lassous, L. Viennot
}

\begin{abstract}
This paper presents a theoretical study on the bandwidth reservation problem for ad hoc networks. The proposed model is based on the spatial reuse and the existence of interferences. We show that in that case, the bandwidth reservation problem is NP-complete and we provide some bounds that compare solutions of the problems derived with greedy heuristics with an optimal one. We conclude with a discussion on the practical aspect of this model and its potential use in a practical protocol.
\end{abstract}

Keywords - Ad hoc networks, bandwidth reservation, interferences.

\section{INTRODUCTION}

This paper deals with a theoretical study on bandwidth reservation problems for mobile ad hoc networks. The ad hoc networks are more and more studied and with the existence of commercial products of wireless network cards, such networks are built in places where wiring is expensive or impossible or when mobility is needed.

The IETF working group MANET concentrates essentially on the routing protocols. Many multicast protocols have also been proposed in the literature. On the other hand, few works have been carried out on the QoS aspects of these networks. This loss of studies on this subject may be explained by the fact that the range of applications offered by the ad hoc networks is not yet well determined, but also by the fact that these networks are seen like networks of small size. In these conditions, the QoS issues do not clearly appear. But the potentiel usage of ad hoc networks in everyday life, their possible or future mix with other large scale networks like Internet or third-generation mobile networks and their capacity to offer high rate could lead to a need of quality of service for such networks.

The QoS issues in ad hoc networks are challenging tasks duc to the dynamic aspects of these networks and their bandwidth constraints ([1]). In the literature, the works concern QoS models (like FQMM presented in [2]), QoS resource reservation signaling (like INSIGNIA proposed in [3], QoS MAC (like MACA/PR in [4]) and QoS routing (see [5], [6] and [7] for three different protocols). Each of these works tries to deal with difficulties inherent to wireless networks like restricted bandwidth, mobility, or broadcast characteristic of radio transmission. But almost none of them studies the impact of interferences on the quality of their protocol. Indeed, in most of the proposed protocols on the subject, each node of the ad hoc networks only acts (i.e. decides to accept or reject or postpone trafic)

K. Bertet: L3i, Université de La Rochelle, FRANCE

C. Chaudet: ENS Lyon, FRANCE

I. Guérin Lassous: INRIA Rhône-Alpes/ENS Lyon, FRANCE

L. Viennot: INRIA Rocquencourt, FRANCE according its local trafic (i.e. the trafic present in the node) without considering the existing trafic in its "neighborood" (i.e. the nodes that interfere with it). However, we think that this "neighbor" trafic can have a strong influence on the behaviour on each node of the network.

For these reasons, we tried to understand the impact of interferences (ratio signal over noise) on the QoS aspects: how to modelize the existence of interferences for the QoS issues, what is the complexity of such a QoS model taking interferences into account, is it practically possible to deal with interferences in QoS protocols to obtain better efficiencies? To begin, we decided to concentrate on the bandwidth reservation problem. The bandwidth reservation can prevent from congestion in the network, congestion that can easily appears in such ad hoc networks. This problem appeared to us to have a direct application whatever the underline use of the ad hoc network. The bandwitdh reservation problem consists either in maximizing the number of transmitting requests or in optimizing the bandwidth, with respect for the available bandwidth and the spatial reuse without interference.

This paper presents a bandwidth reservation model that takes into account the existence of interferences. It is based on the spatial reuse in radio networks. We study the complexity of this model and show that even for simple instances of this model the bandwidth reservation problem is NP-complete. As it is an ongoing research, we finish this paper with remarks and questions on the practical use of the proposed model: how this model can be integrated in an actual protocol.

\section{A simple MODEL OF THE SPATIAL REUSE}

This section presents a simple model of the spatial reuse in radio networks. For a detailed study on the spatial reuse topic for wireless LANs, see [8].

The spatial reuse is the possibility to have two simultaneous communications without interference if they are far enough from each other. We study here the most simple physical propagation model where a receiver put at a distance $r$ from a radio source with a transmitted power $P$ receives a signal with a power $P / r^{\alpha}$. In open space $\alpha=2$, whereas in urban space the GSM propagation models assume $3 \leq \alpha \leq 4$ [9].

Assume that the communications scope is bounded by $R$ (some hundred meters in open space or some dozen meters indoor for wireless LANs). We impose the following local constraint: a node must not transmit if another node within a distance less than $D$ is transmitting. We are going to evaluate the lowest $D$ that ensures that no communica- 
tion is jammed under this condition.
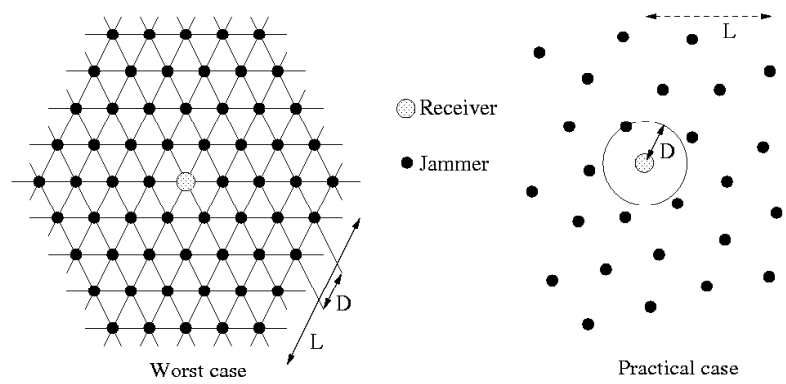

Fig. 1. Two situations where the transmitters are within a distance $D$ from each other, with $L$ the distance of the jammer the furthest in the network. On the left, "a worst case", on the right "a practical situation".

In this paper, we only give a rough estimate of $\frac{D}{R}$ to simply justify the model. We consider what seems to us a worst case: the communication is realized in the center of a trianglular mesh of step $D$ of transmitters, as illustrated in Figure 1. All the nodes are supposed to transmit with the same power. To ensure that a communication is not jammed, the ratio signal over noise has to be higher than a constant $Q$ (classicaly equal to 10). A sufficient condition is then:

$$
\frac{P}{R^{\alpha}} \geq Q B \quad \text { where } \quad B=\sum_{b \text { tansmitter }} \frac{P}{\operatorname{dist}(b)^{\alpha}}
$$

Let $H_{n}$ denotes the hexagon with transmitters at distance $n D$ from the source transmitter (there are $6 n$ such transmitters and their distance is included between $n D \sqrt{3} / 2$ and $n D$ ). If we note $L$ the distance from the transmitter to the furthest node in the network and in grouping the transmitters by concentric hexagons, we have:

$$
\begin{gathered}
B=\sum_{n=1}^{L / D} \sum_{b \in H_{n}} \frac{P}{\operatorname{dist}(b)^{\alpha}} \leq \sum_{n=1}^{L / D} 6 n \frac{P}{(n D \sqrt{3} / 2)^{\alpha}} \\
B \leq \frac{6 P}{(D \sqrt{3} / 2)^{\alpha}} \zeta(\alpha-1)
\end{gathered}
$$

where $\zeta(s)=\sum_{n=1}^{\infty} \frac{1}{n^{s}}$ is the famous Riemann zeta function. Therefore the communication is not jammed for:

$$
\frac{D}{R} \geq \frac{2}{\sqrt{3}}(6 Q \zeta(\alpha-1))^{1 / \alpha}
$$

Figure 2(a) gives an idea of the value of this function (which diverges for $\alpha=2$ ). For $\alpha=3$, a ratio $D / R$ greater than 5.34 is sufficient, or greater than 3.37 for $\alpha=4$.

For $\alpha=2$, it is easy to deduce the following condition :

$$
\frac{D}{R} \geq \frac{2}{\sqrt{3}}(6 Q(1+\ln (L / D)))^{1 / 2}
$$

Figure 2(b) gives the values of $D$ for which the inegality is true.

From this model, we can deduce the following bandwidth reservation scheme:

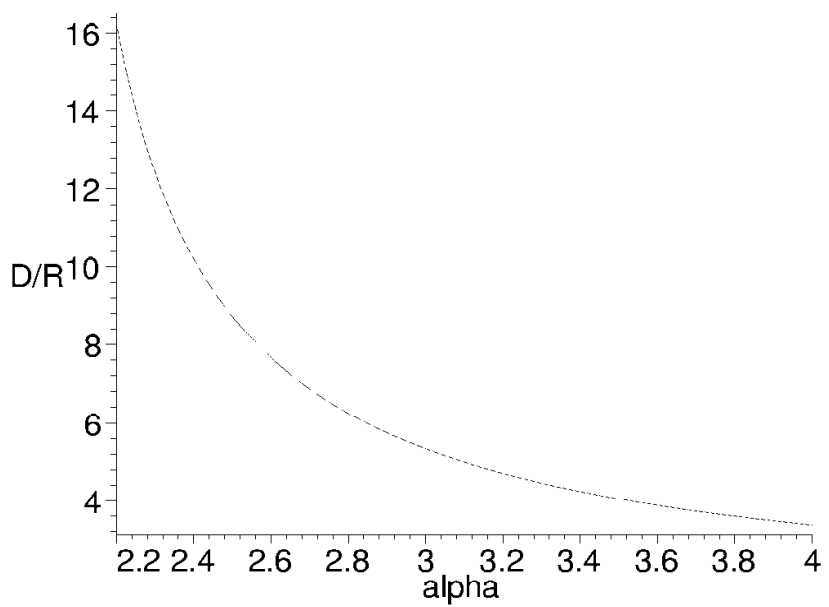

(a) A ratio $D / R$ assuring a lack of interferences according to the attenuation coefficient of power $\alpha$ with $Q=10$.

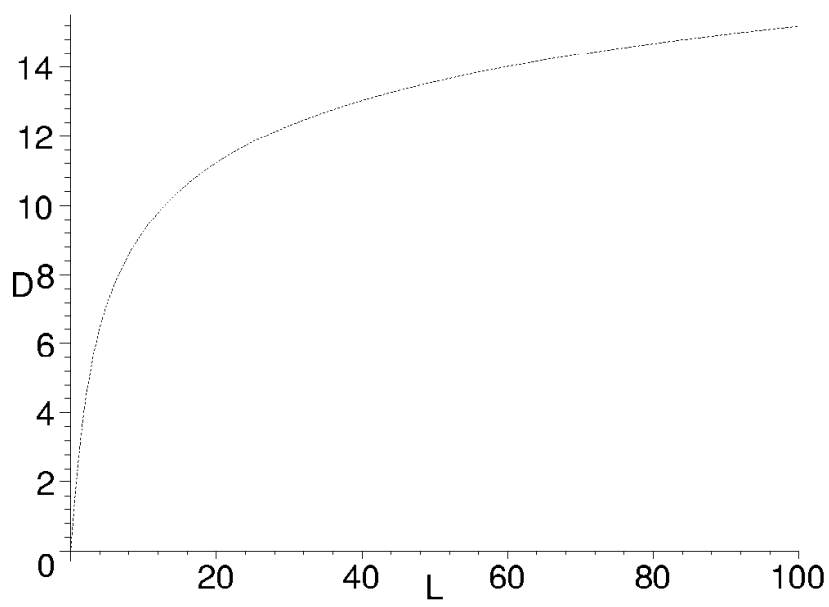

(b) A distance $D$ assuring a lack of interferences according to the radius $L$ of the network with $\alpha=2, R=1$ and $Q=10$.

Fig. 2. $D / R$ according $\alpha$ and $D$ according $L$ for $\alpha=2$.

1. For each node $u$, its interference area $s_{u}$ is defined as being the set of nodes at distance at most $D$ from $u$.

2. Each node $u$ is allowed to reserve a bandwidth $c(u)$ if for all nodes $v$ in the network, the sum of the reservations of bandwidth of the nodes of $s_{v}$ does not exceed the available bandwidth of $s_{v} b\left(s_{v}\right)$. ( $\forall v$ nodes $\left.\sum_{i \in s_{v}} c(i) \leq b\left(s_{v}\right)\right)$

Due to spatial reuse, the available bandwidth can be different from one interference area to one other. Note also than two different nodes can have the same interference area.

If a route (longer than one hop) is established between two nodes $u$ and $v$ in the network, then the request $c(u)$ of node $u$ has to be accepted on the whole route between $u$ and $v$, i.e. accepted on all the nodes of the route. 


\section{TheORETICAL COMPLEXITy OF THE PROPOSED BANDWIDTH RESERVATION SCHEME}

In this section, we theoretically analyse the bandwidth reservation model proposed in Section II. In fact, it is easy to see that this scheme is a sample of the famous 01 multidimensional knapsack. This problem, NP-complete, can be expressed as follows (see $[10],[11],[12],[13]$ for some heuristics):

$$
\sum_{i=1}^{n} a_{j i} x_{i} \leq b_{j}, \forall j \in[1, m], \quad \text { to maximize } \sum_{i=1}^{n} c_{i} x_{i}
$$

where $\forall i \in[1, n]$ and $\forall j \in[1, m] x_{i} \in\{0,1\}$, and $b_{j}, c_{i}$ et $a_{j i}$ are positive integers.

In the framework of our bandwidth reservation scheme: $n$ is the number of nodes in the network and $m$ the number of interference areas (as mentionned previously two nodes can have the same interference area); $\forall j \in[1, m], b_{j} \leq B$ where $b_{j}$ is the available bandwidth in area $s_{j}$ and $B$ is the maximum bandwidth of the network; $a_{j i}=c(i)$ if node $i \in s_{j}, a_{j i}=0$ otherwise; $x_{i}=1$ if the request $c(i)$ of node $i$ is accepted, $x_{i}=0$ otherwise. If the aim is to use the maximum of bandwidth, then $\forall i \in[1, n], c_{i}=c(i)$. If the aim is to satisfy the maximal number of requests then $\forall i \in[1, n], c_{i}=1$.

Our bandwidth reservation problem is a simplified sample of the 0-1 multidimensional knapsack. The radio networks context can simplify the problem. For instance, we can consider that each node of the network belongs to a restricted number of interference areas or that each interference area contains a restricted number of nodes. Moreover, unlike the general problem of $0-1$ multidimensional knapsack where it is assumed that $m$ is small (of the order of ten) and $n$ is large (more than one hundred), in our problem, $n$ and $m$ are of the same order and can be equal to one hundred. These remarks justify to study the complexity of the bandwidth reservation problem and its simplified versions (do they remain NP-complete or not?).

In [14], we show that even the simple instance where each node belongs to a restricted number of interference areas, each interference area has a restricted number of nodes, each node has a unit request (of $1 \mathrm{Mbits} / \mathrm{s}$ if $1 \mathrm{Mbits} / \mathrm{s}$ is the unit) and each interference area has an available bandwidth of 1 Mbits/s, remains NP-complete. See [14], for a detailed description of the proof based on the reduction of the maximum independent set problem. Therefore our bandwidth reservation model is NP-complete.

Many heuristics for the 0-1 multidimensional knapsack problem have been proposed, but the required times to obtain an approximate solution are too long to be used in the bandwidth reservation protocols for mobile ad hoc networks. We think that greedy solutions are probably the most adequate solutions for such networks. We can provide several approximation results for greedy heuristics. We omit the proofs of these results in this paper, but some can be found in [14], others can be easily deduced.
We note $A^{*}$ an optimal solution of the bandwidth reservation scheme and $A$ a greedy solution. $\left|A^{*}\right|(|A|$ resp. $)$ is the size of the optimal solution (the greedy solution resp.). We assume that the node $i$ belongs to $\operatorname{deg}(i)$ interference arcas. We have the following results:

1. If the requests are handled by decreasing values, then $\sum_{i \in A^{*}} c(i) \leq 2 \sum_{i \in A} c(i) \operatorname{deg}(i)$. If moreover, each node belongs to at most $\Delta$ interference areas, we can deduce that $\left|A^{*}\right| \leq 2 \Delta|A|$.

2. If all the requests have a unit cost, then $\sum_{i \in A^{*}} c(i) \leq$ $\sum_{i \in A} c(i) \operatorname{deg}(i)$. If moreover, each node belongs to at most $\Delta$ interference areas, we can deduce that $\left|A^{*}\right| \leq \Delta|A|$.

3 . Whatever the greedy solution we may choose, if each node belongs to at most $\Delta$ interference areas and that each interference area contains at most $\Delta$ nodes, then $\left|A^{*}\right| \leq$ $\Delta(\Delta-1)|A|$. (Note that this case represents the networks with symmetrical links and thus if a node $u$ belongs to an interference area $s_{v}$ then node $v$ belongs to the interference area $s_{u}$.)

$\sum_{i \in A^{*}} c(i)$ represents the maximum use of the bandwidth, whereas $\sum_{i \in A} c(i)$ represents the use of the bandwidth with a greedy heuristic. Intuitively, we see that if the network is dense, the nodes belong to many interference areas and that may affect the quality of the greedy solutions compared to the optimal one (the obtained solution may be at a factor $2 \Delta$ from optimality with a heuristic on the order of the requests). On the other hand, if the network is sparse, the greedy solutions may be close to the optimal solution and thus provide efficient results. Note also that we obtain a better upper bound with the heuristic on the order of the requests (point 1.) than in the general case (point 3.)

\section{REMARKS AND QUESTIONS: HOW TO EXTEND THE PROPOSED MODEL TO AD HOC PROTOCOLS}

We have proposed so far a theoretical bandwidth reservation scheme based on the existence of interferences. We showed that this scheme is NP-complete. We also deduced some upper bounds comparing the optimal solution to the bandwidth reservation problem and simple greedy solutions. These upper bounds show that for some architectures of ad hoc networks, the greedy solutions may be close to the optimal one. These results are only of theoretical interest and we are working on the practical use of this model. We have now to face with several questions in a practical setting:

1. How can each node $u$ determine its interference area, i.e. the nodes that interfere with it?

2. For each node $u$ of the network, is it possible to know the bandwith reservation of each node belonging to its interference area $s_{u}$ without a too large overhead?

3 . Is it possible to distribute this model (that requires a whole knowledge of the network) over the nodes (that will have a local knowledge), knowing that the choice of a node can be influenced by the choice of any other node of the network?

At present, we think that the knowledge of the interference area for each node might be too much to implement 
in a protocol. In a first step, we only consider restricted interference areas. For each node, each interference area contains the one-hop neighbors or two-hop neigbors. We want to check if this knowledge enhances the quality of the bandwidth reservation or not (is there less congestion?; Have the accepted packets a better rate than in the best effort way?; Are there many rejected or postponed packets?). We have two goals in mind: to add this notion of interference areas to reactive protocols to compute routes with good properties (sufficient bandwidth available) and to use the hello packets and the construction of the routing table to carry the necessary information on the interference areas in the proactive protocols. We are currently elaborating these ideas in QoS resource reservation signaling.

\section{REFERENCES}

[1] M. S. Scorson, "Issues in Supporting Quality of Service in Mobile Ad Hoc Networks," in IFIP 5th Int. Workshop on Quality of Service, May 1997.

[2] H. Xiao, W. K. G. Seah, A. Lo, and K. C. Chua, "A Flexible Quality of Service Model for Mobile Ad-Hoc Networks," in IEEE VTC2000-spring, Tokio, Japan, May 2000.

[3] G-S. Ahn, A. T. Campbell, S-B. Lee, and X. Zhang, "draft-ietfmanet-insignia-01.txt," Internet Draft, October 1999.

[4] C. R. Lin and M. Gerla, "MACA/PR: An Asynchronous Multimedia Multihp Wireless Network," in IEEE INFOCOM'g7, 1997.

[5] P. Sinha, R. Sivakumar, and V. Bharghavan, "CEDAR: a CoreExtraction Distributed Ad hoc Routing Algorithm," in IEED Infocom'99, New York, March 1999.

[6] S. Chan and K. Nahrstedt, "Distributed Quality-of-Service Routing in Ad-Hoc Networks," IEEE Journal on Special Areas in Communications, vol. 17, no. 8, August 1999.

[7] R. Lin and J. S. Liu, "QoS Routing in Ad Hoc Wireless Networks," IEEE Journal on Special Areas in Communications, vol. 17, no. 8, August 1999.

[8] S. Tabbane, Réseaux Mobiles, HERMES, 1997.

[9] L. Lebris, Allocation de Ressources Radio, Ph.D. thesis, Université Paris 7, 1999.

[10] A. S. Manne and H. M. Markowitz, "On the solution of discrete programming problems," Econometrica, vol. 25, pp. 84-110, 1957.

[11] R. Loulou and E. Michaelides, "New Greedy-like Heuristics for the Multidimensional 0-1 Knapsack Problem," Operations Research, vol. 27, no. 6, pp. 1101-1114, 1979.

[12] J. S. Lee and M. Guignard, "An approximate algorithm for multidimensional zero-one knapsack problems - a parametric approach," Management Science, vol. 34, no. 3, pp. 402-410, 1988.

[13] A. Fréville and G. Plateau, "An efficient preprocessing procedure for the multidimensional 0-1 knapsack problem," Discrete Applied Mathematics, vol. 49, pp. 189-212, 1994.

[14] K. Bertet, I. Guérin-Lassous, and L. Viennot, "Un premier pas vers la réservation de bande passante," in Actes d'Algo Tel'2000, Mai 2000.

[15] S. Martello and P. Toth, Knapsack Problems, Wiley, 1990.

[16] I. H. Osman and J. P. Kelly, Eds., META-HEURISTICS: Teory and Applications, Kluwer Academic Publishers, 1996.

[17] M. R. Garey and D. S. Johnson, Computers and Intractability - A Guide to the Theory of NP-Completeness, Freeman, 1979.

[18] W.C.Y. Lee, Mobile Cellular Telecommunications, McGrawHill, 2nd edition, 1995. 